\title{
Continuité des rites : le nombril des églises et le placenta des hommes en Polynésie orientale
}

\section{Bruno Saura}

\section{(2) OpenEdition \\ 1 Journals}

Édition électronique

URL : http://journals.openedition.org/jso/1171

DOI : $10.4000 /$ jso. 1171

ISSN : $1760-7256$

\section{Éditeur}

Société des océanistes

\section{Édition imprimée}

Date de publication : 1 juin 2003

Pagination : 39-52

ISSN : 0300-953x

\section{Référence électronique}

Bruno Saura, «Continuité des rites : le nombril des églises et le placenta des hommes en Polynésie orientale », Journal de la Société des Océanistes [En ligne], 116 | Année 2003-1, mis en ligne le 26 mai 2008, consulté le 02 mai 2019. URL : http://journals.openedition.org/jso/1171 ; DOI : 10.4000/ jso. 1171

(C) Tous droits réservés 


\section{Continuité des rites : le nombril des églises et le placenta des hommes en Polynésie orientale}

par

Bruno SAURA *

\section{RÉSUMÉ}

Le rituel chrétien de pose de la première pierre d'une église ou d'un temple revêt en Polynésie orientale une importance symbolique particulière. Il répond en écho à deux traditions autochtones de fondation: l'enterrement du placenta d'un nouveau-né ainsi que la conservation de son cordon ombilical. Cette mise en perspective n'est pas simplement le fait de l'observateur extérieur. Il existe en effet, pour ceux qui les vivent, des correspondances entre ces rites. Bien qu'appartenant à des séquences indépendantes, ils s'influencent réciproquement, se répondant tant dans leur logique que par la nature des objets qu'ils engagent en commun.

MoTS-CLÉS : rituel, placenta, cordon ombilical, fondation, première pierre.

Il est un rituel chrétien, sans lien apparent avec les traditions polynésiennes, qui fait à Tahiti l'objet d'un investissement social important: celui de la pose de la première pierre d'une église ou d'un temple. À cette occasion, chaque fidèle apporte un petit objet personnel qui sera enfermé dans un bocal, lui-même scellé dans les fondations de l'édifice. Après avoir décrit ce rituel, nous chercherons à expliquer son importance symbolique et les raisons du succès de sa perpétuation en faisant observer qu'il répond en écho à deux autres rites de fondation, eux d'origine polynésienne : ceux liés à l'enterrement ou à la conservation du placenta d'un nouveau-né

\begin{abstract}
The Christian ritual constisting in laying the foundation stone of a temple or a church bears in Eastern Polynesia a deep symbolic signification. As a matter of fact, it echos back two autochtoneous traditions of foundation still very observed, the burial of the infant's placenta, and the conservation of its umbilical cord. These correspondances are not only a really for the analyst : as will be shown in this article, the rituals do interfere mutually, both in their logic and through the objects involved in its accomplishment.
\end{abstract}

KEYwORDS : ritual, placenta, umbilical cord, foundation rites, foundation stone.

ainsi que ceux relatifs à son cordon ombilical. On s'apercevra que cette mise en parallèle n'est pas simplement le fait de l'observateur qui analyse les rituels mais qu'il y a bien, pour ceux qui le vivent, correspondance et interrelation - même si elles sont assez largement inconscientes - entre ces rituels. Bien qu'appartenant à des séquences indépendantes (d'un côté, naissance d'une personne; de l'autre, construction d'une maison collective), ils se répondent en effet, tant dans leur logique que par la nature même des objets qu'ils engagent en commun.

\footnotetext{
* Université de la Polynésie Française, B.P. 6570 Faaa Aéroport. Tahiti. Polynésie française. email : brunosaura@yahoo.fr
} 


\section{La pose de la pierre de fondation (niu)}

Nous commencerons par reconstituer le rituel de pose de la première pierre (dite niu) à la fois sur la base d'observations de terrain et à l'aide des comptes rendus rédigés en tahitien et publiés dans les chroniques de la Mission protestante locale dans les années 1940-1950 (journal Ve'a porotetani : Le lien protestant) ${ }^{1}$.

Cette tradition concerne toutes les Églises chrétiennes de Polynésie française. Toutefois, la religion protestante étant la plus ancienne et la plus répandue dans l'ensemble culturel tahitien (îles-du-Vent, îles-sous-le-Vent et les îles Australes), nous nous intéresserons tout particulièrement à l'Église protestante locale. Nous préciserons également que le rituel de la pose d'un niu comprenant un bocal n'existe pas dans la construction des maisons privées de type non religieux.

Chez les protestants, la première pierre peut être soit celle d'un temple (fare purera'a maison de prière ou hiero, terme plus érudit, du grec hieros) soit celle d'une maison de réunion (fare putuputura'a) ou d'une maison paroissiale (fare paroita) dans lesquelles se déroulent des activités cultuelles ainsi que des activités religieuses plus ordinaires (répétitions de chants, etc.).

Plusieurs termes sont utilisés de manière assez interchangeable pour désigner cette première pierre. Ils appartiennent au vocabulaire tahitien pré-chrétien et s'appliquaient autrefois à la construction des marae ou temples traditionnels. Les missionnaires ayant traduit la Bible en tahitien au début du XIX ${ }^{\text {ème }}$ siècle ont choisi d'en conserver certains pour rendre la notion de 'pierre angulaire' ou de 'pierre d'angle' (parfois, 'tête de l'angle') qui figure dans l'Ancien Testament (Esaie $28: 16$; Psaumes $118: 22$ ) et le Nouveau Testament (I Pierre $2: 4-6$; Ephésiens $2: 19-22$, etc.) ${ }^{2}$.

Cette pierre est dite en tahitien 'ófa' $i$-tihi pierre (de) fondation - et, moins souvent, 'ófa' $i$ poro - pierre (d') angle -; On parle aussi de 'ófa' $i$-niu ${ }^{3}$ - pierre d'ancrage, pierre pivot, pierre de fondement -, le terme niu (base/ancrage), à la fois nom et verbe, étant lui aussi souvent employé en lieu et place de 'ófa'i-tihi.

Quant au rituel de pose de la première pierre, il est tantôt intitulé dans les journaux protestants Niuraa 'ofai-tihi 'ancrage (de la) pierre-angu- laire/de fondement', tantôt Haamauraa i te 'ofaitihi 'installation/fixation/scellement de la pierreangulaire/de fondement' (voirVe'a porotetani, juin-juillet $1949: 5$ ).

Bien que très largement utilisé, ce terme niu est ambigu car il peut servir à désigner soit une partie de l'édifice plus large que la première pierre proprement dite soit, à l'inverse, le seul contenu de celle-ci, et non la pierre en tant que contenant.

\section{Les deux sens du terme niu, (pierre de) fondation.}

$\mathrm{Au}$ sens premier, extensif, niu s'applique à l'ensemble de la fondation du bâtiment, comme l'atteste ce récit de la construction du temple protestant de Rangiroa, en 1949 : « Les fondations - te niu - du temple étaient déjà achevées [lorsque] la pose de la pierre d'angle - ofai tihi eut lieu le 14 mai. Les travaux se sont déroulés ainsi : les fondations ont débuté le 4 mai [...] et ont été terminées le 9 . Ces fondations - niu - font 49 pieds et 6 inches de long, pour 28 pieds de large » (Ve'a porotetani, juin-juillet 1949: 6).

Ici, niu est équivalent de papa, terme traditionnel signifiant base, socle (avec une dimension toutefois plus étendue ou horizontale pour le terme papa, tandis que niu, qui signifie également socle/fondation, appelle un développement vertical, une élévation).

On comprend donc que l'emploi du terme niu pour désigner la seule 'première pierre' d'un temple relève de la métonymie puisqu'il désigne aussi l'ensemble de la fondation de l'édifice.

Il est par conséquent tout à fait possible que cette 'première pierre' n'ait pas véritablement été la première posée lors de la construction de la dalle ou de ses murs de soutènement. Confirmation en est donnée à travers le récit de la pose de la première pierre du temple de Pūaoa à Huahine, toujours en 1949. Lors de la cérémonie, «... les murs étaient déjà hauts ; mais on distinguait bien la cavité servant à recevoir le bocal - mohina - dans le mur, du côté intérieur » (Ve'a porotetani, juin-juillet 1949: 5). Cette 'cavité destinée à recevoir le bocal' n'est donc pas exactement une pierre, mais plutôt une cache, une sorte de coffre ou coffrage en ciment et en pierre, bien qu'elle soit aussi appelée dans d'autres textes 'ofai-tihi (première pierre) ou 'ofai-niu (pierre-fondation). Quant à la cérémo-

1. La pluralité des graphies de la langue tahitienne explique que selon les sources citées, un même terme puisse être écrit de différentes manières, s'agissant surtout de la notation de la glottale et de la longueur des voyelles.

2. Même s'ils sont très attachés à la perpétuation de ce rite, les dirigeants de l'Église protestante tahitienne rappellent dans le manuel officiel de liturgie de l'E.E.P.F. (1991: 216) qu'il «n'est pas d'autre fondation (niu) nécessaire à l'homme (de bâtir/d'ancrer) que celle déjà existante qu'est le Christ ».

3. Véa porotetani (décembre 1949, p. 3), à propos de la maison de réunion paroissiale Siona-Tapu de Pape'ete. 
nie de niura'a 'ofai-tihi (ancrage de la première pierre), elle consiste davantage ici à donner un contenu à cette pierre qu'à la poser.

Il arrive fréquemment en effet, dans les conversations tahitiennes, que le terme niu soit utilisé pour désigner le bocal placé dans la cavité d'un temple, et non l'ensemble de la fondation. Le contenu de cette cavité est alors perçu, en quelque sorte, comme le noyau/cœur/pivot/fondement (niu) symbolique du plus grand niu qu'est la dalle de fondation (niu ou papa) de l'édifice.

\section{Les objets transmis}

Outre un bocal en verre d'assez grande taille - en général, au moins 40 centimètres de hauteur -, la cache dite première pierre contient toujours une Bible (en langue tahitienne) et parfois d'autres objets trop volumineux pour être introduits dans le bocal ${ }^{4}$. À l'intérieur de celui-ci sont placés des pièces de monnaie et des petits colliers ou bijoux, parfois en or, offerts par les paroissiens ; certains fidèles nous ont relaté avoir déposé dans le bocal de leur temple un petit message pour leurs descendants et les membres de la communauté qui le liraient un jour, ou encore, une petite pirogue miniature qu'ils avaient fabriquée, etc.

À ces menus objets s'ajoute un texte manuscrit mentionnant officiellement la date de la pose du niu, le nom du responsable des travaux de construction (souvent à la tête d'un groupe de fidèles bénévoles), le nom du pasteur, des diacres et des membres des autorités, feia mana 'gens (de) pouvoir', présents à la cérémonie. Ce texte est la plupart du temps enroulé comme un parchemin, un double en étant conservé au siège de la Mission protestante (devenue en 1963 Église Évangélique de Polynésie française) à Pape'ete. Parfois, c'est sur une feuille de métal que sont inscrits ces noms. L'utilisation d'un bocal en verre, hermétiquement fermé pour que son contenu soit à l'abri des agressions du temps (moisissure, etc.), et le fait que la plupart des objets rassemblés soient en matériaux durables, témoignent de l'importance accordée à la transmission sur une longue durée de ce précieux legs qu'est le niu 5 .

D'une manière plus théorique, la raison de l'introduction de pièces de monnaie dans les fondations d'un édifice religieux n'est pas sans nous rappeler quelques pages classiques de l'Essai sur le sacrifice de Marcel Mauss, dont Rogu Dragan (1999: 49) a récemment souligné les limites s'agissant précisément de rendre compte du choix de cet objet qu'est la monnaie: Mauss posait que l'enterrement d'une victime dans les fondations d'un édifice pouvait servir à la fabrication d'un esprit gardien du lieu, ou bien à se concilier les faveurs d'un « génie du sol » préexistant qui aurait été « lésé par les travaux » (1969, III : 271). Ces explications laissent notamment de côté les cas où le sacrifice n'est qu'un « simulacre », principalement lors des rites d'enfouissement de pièces de monnaie dans une fondation : « Le point capital de la théorie de Mauss était qu'au cours du sacrifice, l'objet sacrifié est détruit, cela étant le seul moyen de l'intégrer au sacré. Il avait raison concernant le sacrifice d'une vie. Mais on ne détruit pas la monnaie, ou l'encens, ou le rouleau avec le nom du fondateur, comme dans les pratiques récentes de fondation. On les protège même, laissant une cachette prévue à ce but dans les fondations de l'édifice » (1999 : 53). Pour Radu Dragan, l'explication est ailleurs : dans la valeur soit directe (intrinsèque), soit indirecte (simplement symbolique) de la monnaie. "Ce qui est [...] intéressant est le fait que l'on n'incorpore pas aux fondations des pièces de grande valeur, tout comme les pièces données au mort : car la monnaie se symbolise ellemême [...]. La monnaie, dénominateur universel de la valeur, arrive ainsi à remplacer la victime. $\mathrm{Si}$ le rite n'est qu'une fausse monnaie, comme pensait Mauss, il serait plus exact de dire que la monnaie est un moyen approprié pour tout rite, et surtout pour le sacrifice» (1999: 53-54, 60-61).

\section{L'emplacement de la 'première pierre' et les cas de pluralité de niu.}

Il reste à nous intéresser au choix du lieu de pose ou de fixation de la pierre de fondation dans le temple. En Polynésie orientale, l'emplacement exact de la 'première-pierre' varie souvent. Bien qu'elle soit dite pierre angulaire, elle ne se trouve pas nécessairement dans un des angles de l'édifice mais parfois près de la porte d'entrée; ou bien sous l'abri de la cloche (fare oe) - qui n'est guère, chez les protestants, située très haut, et toujours près de l'entrée du temple - ; ou encore sous la chaire du pasteur (purupiti), elle-même située à l'avant du temple, tantôt sur le côté,

4. Voir par exemple, pour la pose du niu du temple Tarema de Maeva à Huahine le samedi 16 mars 1991, La dépêche de Tahiti (22 mars $1991: 40)$.

5. Quant à la Bible, elle est par essence ensemble de " paroles solides » (parau pa'ari), c'est-à-dire, de traditions, et " parole vraie/fixe » (parau mau). Sa place dans le niu est toute trouvée puisque c'est sur cette parole qu'est fondée/ancrée (niuhia) l'Église chrétienne. 
tantôt au milieu de celui-ci face à la porte d'entrée. Plusieurs informateurs s'accordent pour dire que la première pierre doit surtout se trouver à l'est, « du côté d'où se lève la lumière ».

Dans le cas d'un édifice démoli et reconstruit, ce qui est fréquent quand on sait le goût des Polynésiens pour la construction des bâtiments religieux, il se peut que le temple ait plusieurs 'ófai-tihi (pierres angulaires) et/ou plusieurs niu (noyaux) ${ }^{6}$.

Ainsi, un compte rendu de 1947 évoque-t-il le haamauraa toru $i$ te ofai-tihi o te fare-pureraa Salema $i$ Vaitoare, soit la «troisième pose/ fixation de la pierre de fondation du temple Salem à Vaito'are » dans l'île de Taha'a, en ces termes : « [Le pasteur] Tuturi fit une prière, après quoi, l'ancienne construction fut dégagée/ découverte, et (l'on vit qu') il y avait deux ofaitihi (pierres de fondation), celle du premier temple et celle du second temple. Ce fut l'Administrateur d'État qui retira les deux bocaux contenus dans chaque pierre de fondation » (Ve'a porotetani, décembre $1947: 5$ ).

Dans le cas où il y aurait plusieurs niu (pierres ou bocaux), deux solutions se présentent, consistant soit à les inclure séparément dans la nouvelle cache, soit à ouvrir les bocaux et à placer leur contenu dans un troisième. Sans anticiper sur les fonctions du niu, on peut d'ores et déjà faire remarquer qu'en cas de démolition d'un temple, il est nécessaire de faire appel à la mémoire collective, en particulier au sein des familles de pasteurs et de diacres, pour retrouver le ou les niu afin de ne pas les endommager lors des travaux.

\section{Le cérémonial de la pose du niu.}

La cérémonie de pose du niu proprement dite voit une alternance de prières, de chants et de discours. Plusieurs pasteurs ou, à défaut, un pasteur et des diacres, se partagent les tâches : prière d'ouverture; sermon sur la base d'un verset biblique; lecture du texte manuscrit qui sera glissé dans le niu; scellement de celui-ci, suivi d'une nouvelle prière.

$\mathrm{Si}$ la construction est entièrement nouvelle, un nom (i'oa) est donné à ce bâtiment (topa 'tombé' ; ma'iri 'glissé, passé') par l'un des offi- ciants ; autrement, il reprend le nom de l'édifice préexistant. Le nom d'un temple ou d'une maison paroissiale est la plupart du temps celui d'un lieu biblique tahitianisé (par exemple, Siona, prononcé Tiona, pour le mont Sion; Betela, prononcé Petera, pour Béthel, etc.), mais il peut s'agir d'un nom doté d'une symbolique plus large : Te Hoa Tumu (L'Ami Véritable), ou Pou Ata (Pilier des nuages) pour certains des édifices ci-dessus mentionnés ${ }^{7}$.

Pour en revenir à l'ambiguïté de l'utilisation des termes souvent interchangeables niu et 'ófaitihi désignant tantôt les fondations entières, tantôt la cache abritant le bocal, tantôt le bocal lui-même, celle-ci peut s'expliquer en raison de la forte dimension symbolique ou de la grande valeur d'abstraction de ces termes. Niu ne désigne pas simplement les 'racines' d'un édifice religieux. Il est aussi l'ancien nom du cocotier à Tahiti et aux Tuamotu ; et l'on sait que, dans ces îles, comme le précise Stimson et Marshall (1964) dans leur dictionnaire des dialectes des Tuamotu, «des poteaux en tronc de cocotier sont fréquemment utilisés dans les fondations des maisons ${ }^{8}$. Niu peut encore s'appliquer à divers objets, désigner le sillage d'un bateau, un guerrier courageux, etc. (voir Davies 1851 : 155). Dans tous les cas, une idée domine, celle de profondeur ou de solidité d'une entreprise, d'un édifice ou bien d'un homme, pilier d'une armée, par exemple.

La polysémie du terme niu (fondement/ ancrage) est également, pour partie, la conséquence de l'acculturation religieuse et matérielle de la Polynésie qui, on va le voir, a entraîné un changement dans la nature des objets placés dans le niu ou constituant le niu. Malgré tout, nous soulignerons surtout les similitudes existant entre le mode d'apparition et, pourrait-on dire, le processus d'engendrement des marae (temples) traditionnels, et la continuité de fondation symbolisée par le niu dans le cas de la reconstruction des édifices chrétiens d'aujourd'hui.

\section{L'engendrement des temples}

S'agissant de la nature des objets placés dans le niu, notons que la circulation régulière de

6. Par exemple, le temple protestant Tiroama de Mata'ura à Tupua'i (Tubuai, îles Australes), dont la construction aurait commencé au début des années 1950, fut inauguré en 1958, restauré en 1985, puis entièrement démoli en 1995 et inauguré à nouveau en 1998. (D'après Teri'inuipe'etautāne'ehu Teinauri et Thomas Hauata, entretien en juillet 1997).

7. Te Hoa tumu pour le fare putuputura'a (maison de réunion) du 'amuira'a (section de la paroisse) Siona-Tapu de Pape'ete (voir Ve'a porotetani, décembre 1949, p. 3) ; Pou Ata pour le temple - qui ne sera finalement jamais inauguré - de Pūaoa à Huahine (voir Ve'a porotetani, juin-juillet $1949: 5$ ).

8. Stimson (1964:332) : "The coconut log is frequently used for a house foundation in present-day Oceania ». Le même auteur indique l'existence d'un terme désuet, Niutākohua, nom d'un cocotier de la légende de Māui et Atarana, et s'appliquant peut-être aussi au cocotier censé se trouver au centre du monde. Ici, niu signifie donc à la fois cocotier et pilier de fondation. 
matériaux en fer et en verre remonte en Polynésie orientale à la fin XVIII $^{\text {ème }}$ siècle. Quant au premier temple de l'ensemble tahitien construit en pierre, il est celui de Papeto'ai à Mo'orea, dont les fondations furent posées en février 1822. Ce bâtiment hexagonal, inauguré en 1827 , est d'ailleurs le plus vieux monument chrétien en pierre de tout le Pacifique. Toutefois, tout prête à penser qu'il a fallu attendre plusieurs décennies plus tard pour qu'un bocal soit systématiquement inclus dans les fondements d'un édifice religieux chrétien.

Dans les premiers édifices chrétiens en Polynésie orientale, la pierre angulaire ou première pierre était véritablement une pierre et non une cache creuse. Le pasteur Grégoire Tumarae, dit Rono, rapporte ainsi (Entretien du 8 juillet 1997) qu'à Vaiuru, dans l'île de Ra'ivavae (Australes), le fare paroita ('maison paroissiale'), comprenait deux niu, deux pierres non creusées : une pierre carrée, 'ófa'i ma'atea, spongieuse comme du corail, et une pierre ronde, de couleur rouge foncé, 'ōfa'i 'ute'ute. L'une paraissait provenir d'une construction plus ancienne - dont on ignore la nature, chrétienne ou non -, et toutes les deux étaient placées près de la porte d'entrée de l'édifice. Dans la même île, le temple du village de Mahanatoa avait pour niu une seule pierre rouge, mais pas non plus de bocal.

\section{Continuité des traditions}

L'idée d'introduire dans les fondations d'un temple un bocal contenant des reliques correspond à une pratique chrétienne assez récente (environ cent cinquante ans) en Polynésie orientale, mais plus ancienne dans l'Occident chrétien. En revanche, l'importance accordée à la pose de la première pierre d'un édifice religieux révèle une préoccupation conjointe des deux ensembles culturels.

Ainsi, dans le Tahiti des temps anciens, la pierre la plus importante d'un marae était son ha' $a i$, dalle de corail ou pierre basaltique correspondant à la branche aînée d'une famille ou chefferie. C'est assis sur cette pierre ou adossé contre elle que siégeait son représentant titulaire.
Évoquant le premier marae supposé avoir été édifié dans l'ensemble tahitien, le marae Vaeāra'i de Ra'iatea, la dernière reine de Tahiti, Marau Ta'aroa (1971:51-52) précise que ce ha'ai était aussi entouré, à droite et à gauche, de quatre pierres, dites terahu.

Si le $h a^{\prime} a i$ formait la pierre centrale du marae, il semble qu'il n'en était pas d'ordinaire la première pierre, comme le suggère la suite du texte, relative au fameux marae Farepu'a de Vaiari (à Tahiti) : «D'après la tradition, après avoir créé Hiti nui - Tahiti -, Ta'aroa posa son pied sur un bloc de corail blanc à Vaiari. [...] De ce bloc de corail blanc fut détachée la pierre qui servit de fondation au temple de Farepua; ainsi que le papa, ou siège royal de ce temple, qui fut appelé Te papa o te toa o Taaroa, le siège de corail de Taaroa » (1971 : 52). Ici, le papa (socle) s'identifie donc au ha'ai.

Dans sa narration des étapes de la construction d'un marae national (1962: 139), Teuira Henry nomme 'ofa'i-faoa ${ }^{9}$ (pierre principale de fondation) la première pierre du marae, précisant que celle-ci était de grande taille. « [...] Une autre pierre appelée $t i^{\prime} a$-va (debout-dominant) était placée dans le coin opposé et ces deux pierres étaient appelées tihi-marae (cornes du marae)' ${ }^{10}$. Établissant elle aussi le parallèle entre les traditions judéo-chrétiennes et celle de Tahiti, Teuira Henry ne manque pas d'ajouter que ces pierres avaient «la même signification que les cornes des Temples juifs » (ibid.).

C'est toujours par "clonage » qu'apparaissaient de nouveaux marae, dont la construction s'organisait autour d'une pierre retirée d'un marae de rattachement antérieur. Il pouvait arriver que ce soit le ha'ai (siège central) qu'emporte le fondateur d'un nouvel édifice, comme le ha'ai de Vaeāra'i à Ra'iatea qui devint la première pierre du prestigieux marae Vai'otaha à Bora Bora (voir Cadousteau 1987 : 8). Mais, logiquement, la fondation d'un marae étant le fait d'une branche cadette qui s'émancipait de son marae familial (en en créant un autre), le ha'ai demeurait le siège des aînés et donc du marae d'origine ; et ce sont d'autres pierres, angulaires ou non, de ce marae, qui étaient prélevées pour servir de commencement à un nouveau marae ${ }^{11}$. Quant

9. Plus loin (1962: 205), elle nomme la cérémonie de pose de cette pierre Faora'a. Les consonnes h et f étant équivalentes en tahitien, il faut lire Haora'a pour la cérémonie et Haoa pour le nom de la pierre de fondation. Voir aussi Davies (1851 : 97-98).

10. On retrouve ici la base du terme 'ôfa'i-tihi désignant aujourd'hui la pierre angulaire.

11. Puis, les nouveaux marae devenaient à leur tour des marae d'origine de nouvelles lignées, et ainsi de suite. Même si logiquement, tous les marae devraient être issus d'un même marae fondateur que certaines traditions présentent comme Vaeāra'i, il reste qu'à l'échelle de chaque île, un marae était également reconnu comme marae central, marae d'origine ou de souche, dit 'ere'ere fenua (socle sombre) ou pū-marae : marae - noyau ( $p \bar{u}$ désignant une origine, un centre ; on retrouvera cette racine dans le terme püfenua désignant le placenta). Pour l'île de Huahine, Émile Hiro (1997 : 30) évoque ainsi le marae Mata'irea (ancien nom de l'île) comme pü marae tumu 'centre/marae/racine' ou 'cœur véritable de(s) marae' de l'île. De la même façon, une chefferie était-elle considérée comme « première » de l'île : ainsi, selon le même auteur, 'Öpoa était le pü mata'eina'a (chefferie 
au terme niu proprement dit, Teuira Henry (1962, p. 140) le traduit par "fondation" et explique qu'il correspondait non pas à la première pierre mais à la première rangée de pierres (premier étage) du marae, c'est-à-dire, à son socle.

Contrairement à la situation contemporaine, il est tout à fait possible que le rituel de la pose de la première pierre n'ait autrefois pas concerné les seuls édifices religieux. Tout d'abord, il faut se garder de percevoir le marae comme un édifice uniquement religieux à l'image d'un temple chrétien. Il existait en effet de nombreuses sortes de marae, ce terme s'appliquant à tout espace, en général une structure lithique sur laquelle se déroulaient des cérémonies, qu'elles soient agricoles, familiales, guerrières, etc. Par ailleurs, les marae étaient construits dans la continuation des résidences royales (ou de chefferie), lesquelles étaient entourées d'habitations de gens de moindre rang disposant également de marae, et ainsi de suite. Si bien qu'il s'avère parfois difficile de distinguer le pavage de certaines maisons de celui des marae placés à proximité immédiate, comme l'ont bien montré les fouilles de Pierre Vérin (1969) pour la « cité royale » de Vitaria à Rurutu (Australes).

Il est possible d'émettre l'hypothèse que, jadis, en Polynésie orientale, l'ensemble des habitations d'importance auraient été pourvues d'une première pierre, un noyau ou 'cœur' $(p \bar{u})$, que seuls les édifices religieux auraient conservé ${ }^{12}$. Et si l'on sait que la plupart des temples chrétiens ont été édifiés, au XIX ${ }^{\mathrm{ème}}$ siècle, non seulement à l'emplacement des marae traditionnels, mais aussi avec les pierres de ceux-ci ${ }^{13}$, on comprend mieux la continuité existant de facto, en dépit de l'idéologie chrétienne véhiculée par les Polynésiens eux-mêmes, entre les marae d'autrefois et les temples ou chapelles d'aujourd'hui. Pourtant, le marae reste dans bien des mémoires lié au souvenir des sacrifices humains qui se déroulaient sur certains d'entre eux, souvenir qui explique le désintérêt présent souvent mêlé de peur qu'inspirent ces sites pré-chrétiens aux habitants de l'ensemble tahitien.

Précisément, au sujet de ces sacrifices humains, la construction des grands marae royaux exigeait l'offrande de victimes. Teuira Henry (1962 : 139) précise que, sous la première des deux 'ôfa' $i$-tihi (pierres de fondation) de chaque marae national, était placé le corps d'un homme sacrifié à l'occasion de la cérémonie de fondation, homme dont l'esprit devait monter la garde. La tradition rapporte aussi que pour l'édification du marae international Taputapuātea de Raiatea, quatre hommes furent enterrés vivants sous chacun des coins du « fameux bloc en pierre blanche [...] appelé Te-papa-tea-ia-ru'ea - le roc blanc de l'investiture - . Pour cette raison, ce bloc de pierre était appelé Te-papa-o-na-maha: le rocher des quatre » (Robineau 1986:94; voir également Ellis 1972 : 218). Lieu de scellement d'alliances guerrières ou de déroulement de fastueux mariages, ce marae de Taputapuātea était aussi, comme quantité d'autres marae, un lieu de dépôt des reliques des morts, glissées sous les pierres ou dans leurs interstices ${ }^{14}$.

Indépendamment de la logique - sacrificielle ou non - de la religion et de l'acte religieux en question ${ }^{15}$, l'idée d'une incorporation des reliques de morts à la structure des édifices religieux, certaines reliques pouvant même leur servir de " cœur symbolique », de soubassement de leur

centre) de Ra'iatea (1997 : 106). Dans une société fondée sur l'aînesse, cette chefferie était logiquement celle du marae originel. Par contre, en matière d'exercice des droits politiques, Teuira Henry (1962:149) précise que « avec la pierre de l'ancien marae, disparaissaient aussi les titres héréditaires qui appartenaient à l'ancien propriétaire, et les nouveaux possesseurs du marae n'avaient plus aucun droit sur ces titres ».

12. C'est ce qu'affirmait le sage de Taha'a, Paimore Tehuitua, rencontré le 4 juin 1997. De manière assez contradictoire, il posait aussi qu'un marae dépourvu de 'ófa'i tihi (pierre de fondation) ne méritait pas le nom de marae (temple) mais seulement celui de pa'epa'e (plate-forme). Il y a contradiction dans la mesure où les maisons sont dites posséder un niu ou 'öfai-tihi, qui est en même temps perçu comme l'apanage du marae.

13. Teuira Henry (1962 : 137) mentionne le cas du petit temple octogonal construit par Platt à l'emplacement exact du marae Tūramarafea de Papeto'ai à Mo'orea. «A la droite de l'église se trouve la fameuse pierre à prières haute de 2 mètres et d'une surface de 30 centimètres carrés » consacrée au dieu 'Oro. Un autre cas célèbre est celui du marae Tainu'u à Tevaito'a dans l'île de Ra'iatea, saisissant parce que la majeure partie de l'édifice traditionnel existe toujours juste derrière le temple chrétien, en bord de mer.

14. D'autres marae, surtout aux Tuamotu, servaient à la consommation des meilleurs poissons et des tortues, dont les restes étaient également enterrés sur place.

15. Commentant diverses coutumes (africaines et occidentales) de fondation d'un village avec enterrement d'une victime dans laquelle était planté un pieu ou un pilier, Radu Dragan (1999 : 27) pose que toute cérémonie de fondation "peut revêtir plusieurs formes, du sacrifice proprement dit à la métaphore [...]. Le pieu est une métaphore du corps, renforcée par le sacrifice sanglant ; mais l'idée de sacrifice lui était déjà incorporée, car occuper un territoire est toujours une 'conquête' impliquant le sacrifice de soi ». Dans le cas polynésien, outre le fait que le marae (hier) et le temple (aujourd'hui) soient un lieu de sacrifice ou de commémoration d'un sacrifice, la construction de ce genre d'édifice représente effectivement un lourd investissement, un sacrifice collectif ; ces deux raisons pourraient expliquer pourquoi la fondation d'un marae comme d'un temple nécessite l'existence d'une pierre d'angle ou d'une cachette de type niu, alors que tel n'est pas (et n'aurait peut-être jamais été) le cas pour une maison ordinaire. 


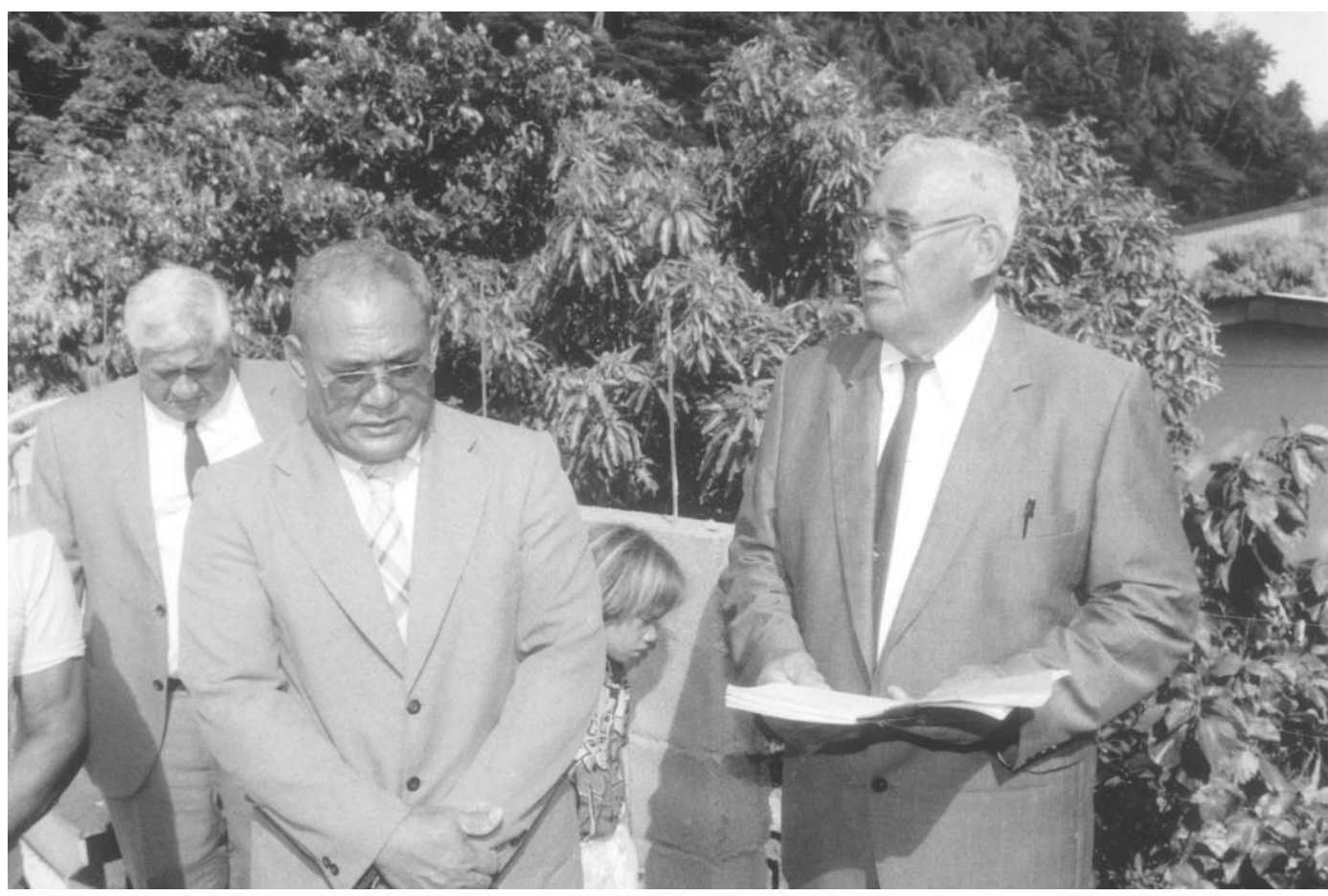

Séquence 1 : Une prière est dite par le pasteur (ici,Terito Tepa) au début de la cérémonie consistant à donner un contenu à la 'première pierre' (niu) d'un temple. Ici, le temple de Maeva à Huahine (îles-sous-le-Vent), dont la pose de la 'première pierre' eut lieu le 16 mars 1991 (les autres photos sont relatives au même événement).

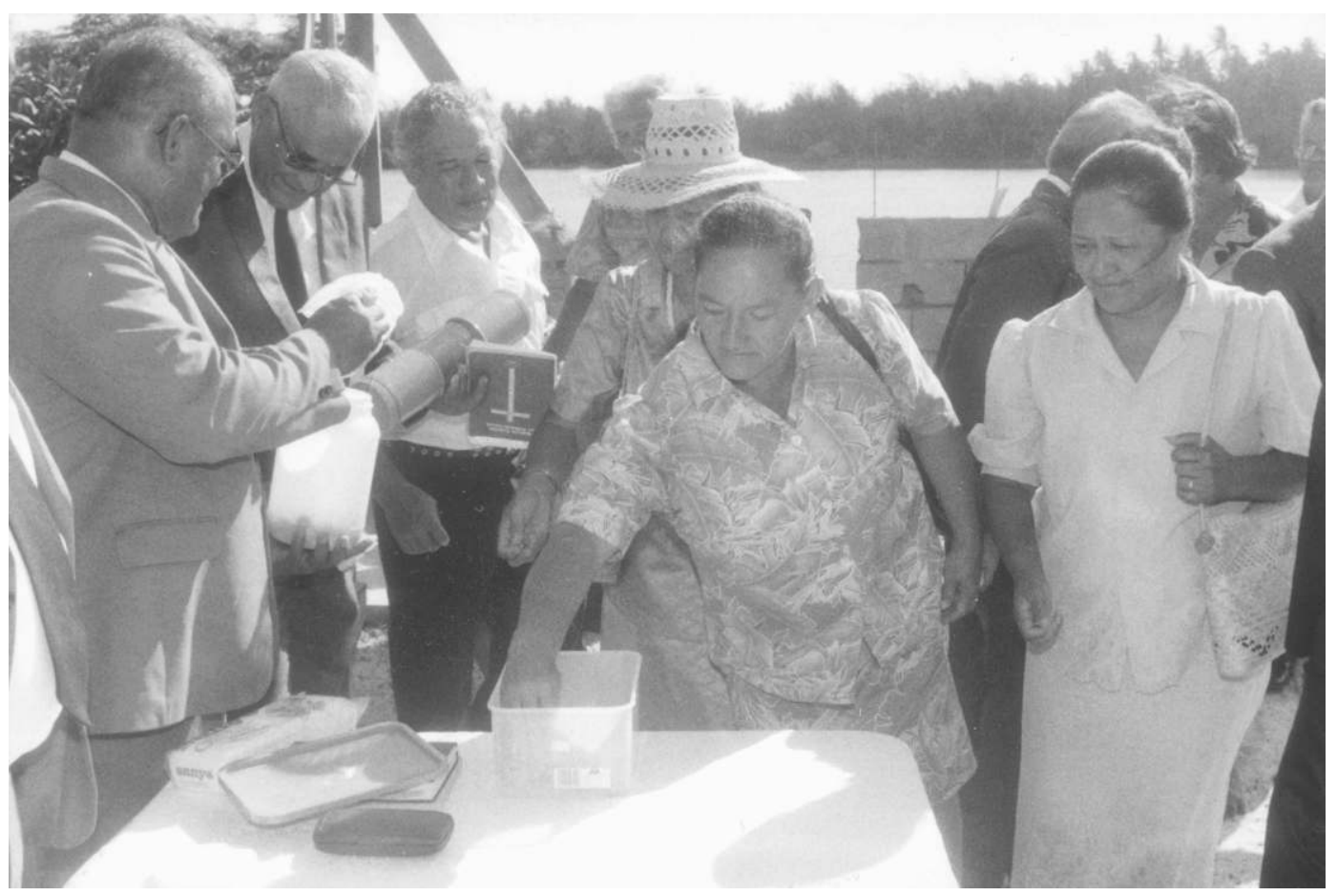

Séquence 2 : Tour à tour, chaque fidèle de la paroisse protestante qui le désire place un petit objet personnel dans une boîte hermétique, sous le regard du pasteur et des diacres. 

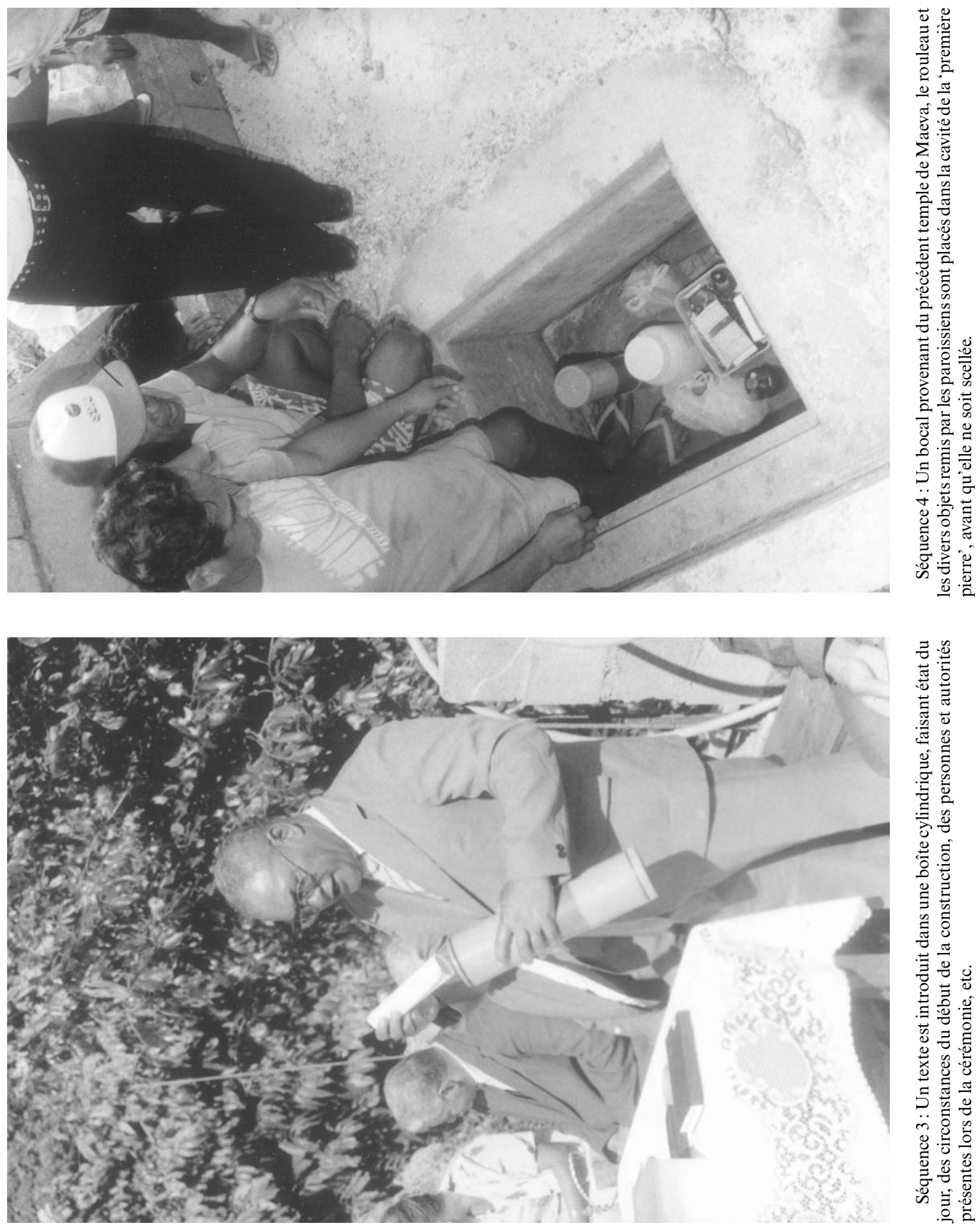


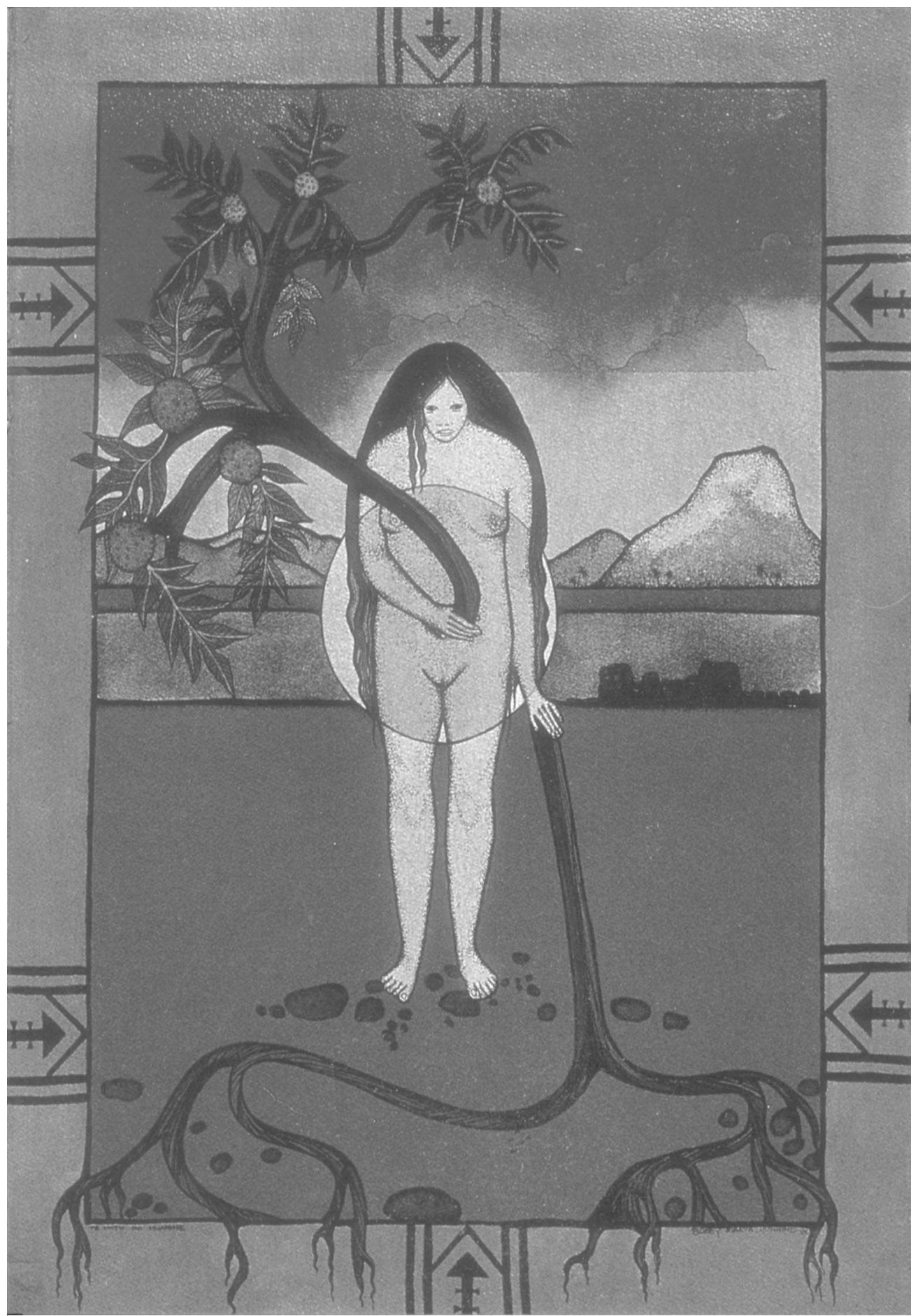

Tableau : « Te hotu o te fenua, Le fruit de la terre »

Ce tableau du peintre hawaïen Bobby Holcomb, peint en 1975, représente une femme polynésienne enceinte, portant en elle un enfant relié par son cordon ombilical à son placenta nourricier dit püfenua en tahitien. Le püfenua, terme qui signifie 'noyau (de) terre', est d'ordinaire enterré auprès d'un arbre lui aussi nourricier. On mesure là la continuité de fructification existant chez les Tahitiens entre le placenta, la terre, les arbres et les hommes. L'arbre à pain représenté sur ce tableau est, dans la tradition, né de la transformation du corps de l'homme Rua-ta'ata, qui s'enterra et prit racine pour sauver sa famille de la disette, sa tête devenant le fruit de l'arbre à pain, dit 'uru.

Le rite d'enterrement du placenta fait partie des rites de fondation très importants chez les Tahitiens, dont celui de pose de la première pierre d'un édifice religieux (hier, marae, aujourd'hui, temple ou église). 
pierre de fondation, ou être placées à l'intérieur de celle-ci, se retrouve ainsi en Polynésie comme dans les anciennes traditions judéo-chrétiennes. Dans celles transmises par l'Église catholique, deux rituels coexistent, qui se rejoignent respectivement et répondent aussi en écho logique aux rituels polynésiens :

- Le premier est la pose de la pierre de fondement d'une église (dans le vocabulaire catholique polynésien: 'ófa'i poro, pierre d'angle) à l'intérieur de laquelle est scellé un bocal contenant des reliques. Ce rite ne fait plus aujourd'hui partie des rites officiels de l'Église romaine, mais il continue à être pratiqué tant en Occident qu'en Polynésie.

- Le second rite, officiel, consiste, lors de la construction d'une église, à placer des reliques qui sont en général d'importance (un fragment d'os, de tissu, de Saint-Pierre Chanel, de SainteThérèse de Lisieux, etc.) à l'intérieur d'une boîte en verre ou en métal glissée dans une cavité dite 'ápo'o o te toe'a (trou des reliques). Cette cavité se situe le plus souvent au pied de l'autel, ou dans sa structure même (Entretien avec l'Archevêque de Pape'ete, Monseigneur Michel Coppenrath, le 4 avril 1997). L'origine de ces deux pratiques remonte au temps du christianisme primitif, époque à laquelle l'eucharistie était célébrée sur le tombeau des martyrs.

Il n'est donc guère difficile d'établir une continuité de logique entre des rituels relevant de traditions et d'époques différentes puisque chaque fois, la signification mise en œuvre est la même, quelle que soit la nature des objets manipulés : la première pierre est symboliquement comme l'embryon d'un corps nouveau, embryon dans lequel les hommes ont soin d'introduire une mémoire, un patrimoine en condensé qui est ensuite enfermé dans le niu. Celui-ci est constitué des reliques des morts d'hier (ossements) ou des attaches des vivants d'aujourd'hui (chaînes, bagues) qui revêtiront à leur tour le caractère de reliques lorsque le contenu de ce niu aura été mis au jour par la génération d'hommes appelés à rebâtir le temple.

On peut aussi faire remarquer combien ces rituels relatifs au corps d'un temple font écho à ceux relatifs aux cycles de la vie d'une personne. Ceux d'ouverture ou de fondation, matérialisation d'une conception ou moment d'une naissance, sont marqués par l'octroi d'un nom. Plus tard, lorsque la croissance/construction de cet édifice sera achevée, son inauguration verra son ouverture au public, pénétration rituelle sur laquelle le vocabulaire tahitien traditionnel - et toujours actuel - est particulièrement explicite.
'Inaugurer' se dit en effet tomo (pénétrer) ou 'āvari, terme construit sur la base de vari désignant la boue et aussi l'écoulement du sang menstruel. L'inauguration ('âvarira'a) d'un bâtiment religieux correspond par conséquent à sa souillure ou 'défloration' symbolique. Mais les fleurs sont appelées à donner des fruits, ce que confirme à nouveau le vocabulaire: 'âvari s'appliquait autrefois à la fin d'un rāhui, c'est-àdire, d'une interdiction (tapu, tabou) de récolte de certains produits de la terre ou de la mer en un temps et un lieu déterminés. Par conséquent, 'āvari signifie à la fois profaner, désacraliser un lieu, et ouvrir au public la récolte d'un champ après que les premiers fruits en aient été offerts au chef.

\section{Le rite et les reliques}

Que les rituels liés à l'élévation d'un temple comme ceux ayant trait à la naissance d'un individu se rejoignent ne saurait nous étonner : il s'agit dans les deux cas de rites de mise en place symbolique d'un ordre, à proprement parler, de rites de fondation. Nous pouvons maintenant aller au-delà et tenter d'établir d'autres correspondances entre la pose de la première pierre d'un temple et le traitement réservé en Polynésie à un type particulier de " reliques » ou de restes « originels » : le placenta d'un enfant, ainsi que la partie du cordon ombilical (pito) attachée à son corps et qui s'en détache quelques jours après la naissance. "L'intuition » de ce rapprochement nous est donnée du fait de la conservation, aujourd'hui, de ce cordon ombilical desséché, à l'intérieur d'un petit bocal, lequel rappelle le bocal compris dans le niu d'un édifice religieux.

Bien qu'il soit lié à un corps vivant (celui du nouveau-né), le cordon ombilical (pito) peut très vite être apparenté à une relique et manipulé comme tel. D'une manière générale, dans un rituel, la nature - ou si l'on veut, l'origine - des objets engagés ne conditionne en effet que partiellement le sens qui leur est donné ; d'où l'existence de « ressemblances de détail entre certains rites de la naissance et certains rites des funérailles » déjà notée par Arnold Van Gennep au début du siècle dernier (1909: 75) dans son ouvrage sur les rites de passage.

Dans le cas polynésien, Alain Babadzan (1983) a précisément bien mis en évidence les similitudes existant autrefois entre le traitement des corps morts embaumés et celui du cordon ombilical, également oint d'huile puis soigneusement conservé. Nous souhaiterions étendre le 
qualificatif de relique au placenta, appelé en tahitien püfenua, ce qui signifie 'noyau/cœur/ centre' (pü) de 'terre' (fenua); si le placenta ne peut véritablement se conserver, de par son caractère très fragile et corruptible, on a montré ailleurs qu'il partageait la plupart du temps avec le pito (cordon) une identité de destination et aussi de signification à l'intérieur des rites de fondation polynésiens ${ }^{16}$.

Pour nous en tenir aux enfants issus des familles de chefs, celles dont les pratiques sociales sont les mieux connues, Marau Ta'aroa (1971 : 60) rapporte qu’à Tahiti, « la naissance (de l'enfant) était annoncée au peuple par les instruments sacrés du marae. Les prêtres devaient enterrer sur le marae le placenta aussitôt après qu'il avait été détaché de l'enfant. Le nombril 17 était aussi enterré sur le marae avec le même cérémonial, les prières, les battements rythmés des tambours sacrés ».

De son côté, Teuira Henry (1962 : 192) précise que le cordon ombilical détaché «était placé, enveloppé dans un morceau de tapa (tissu fait d'écorce battue) parfumé et placé par le paia (prêtre) dans un joint de bambou que l'on introduisait dans un autre plus grand formant ainsi une boîte hermétique ; cette boîte était placée dans la cache du marae ancestral. Cette relique était considérée comme l'essence de l'enfant $»$. Or, essence se dit en tahitien iho, soit aussi un des deux termes (avec pito) qui en Nouvelle-Zélande désignait le cordon ombilical.

La pratique consistant à introduire une boîte contenant ce type de relique " essentielle», à l'intérieur de la structure du marae (temple) rappelle exactement celle ayant lieu lors de la pose de la première pierre (niu), «cœur» ou « essence » des temples modernes. Cette corres- pondance va au-delà d'une relation d'analogie ou homologie de rapports par laquelle on pourrait dire que le cordon ombilical est à l'enfant ce que le niu est au marae/temple : son essence, la marque de son origine, son premier ancrage. Ici, non seulement les deux rituels se répondent logiquement, mais ils vont à la rencontre l'un de l'autre dans la mesure où le premier ancrage de l'enfant vient trouver sa place dans la structure d'un temple sous la première pierre duquel se trouvent d'autres reliques de fondation (dans le cas du marae traditionnel, celles des morts offerts en sacrifice). L'identité du rituel entre la pose de la première pierre et l'enfouissement du cordon débouche sur une identité de signification des objets engagés. C'est en effet le même terme iho, essence, s'appliquant, on l'a vu, au cordon ombilical, qui désignait la pierre « enterrée sous chaque poteau d'autel - destinés à l'étalage des offrandes de nourriture et sacrifices (pierre) enveloppée d'une ou deux feuilles du miro ${ }^{18}$ le plus sacré, appelée iho de l'autel sacré $\gg{ }^{19}$.

Même dans le cas où le cordon ombilical et le placenta auraient eu pour destination un autre endroit que le marae, cette coïncidence entre les rites, qui affecte la signification des objets, demeure.

Il arrivait en effet, et la pratique s'est amplifiée à Tahiti avec la disparition des marae, que le placenta soit mis en terre dans la cour d'une habitation, auprès d'un arbre ; ou inversement, qu'un nouvel arbre soit enterré sur ce placenta, afin de nourrir ses racines de la substance ayant nourri l'enfant. Le placenta et le cordon pouvaient aussi être enterrés à l'intérieur des habitations... dans le sol desquelles étaient parfois mis en terre les corps des défunts ${ }^{20}$.

16. Voir Saura (2002). Le présent article relatif au cœur des églises s'inscrit dans un ensemble de trois textes que nous avons consacrés à l'enterrement du placenta en Polynésie française. Dans un premier travail (2000), était mesurée la perpétuation de cette pratique au moyen d'une enquête à la maternité de Pape'ete, et également abordé le sens que les Tahitiens d'aujourd'hui lui donnent, notamment à travers un exemple de réinterprétation identitaire très contemporain. Un second article (2002) expliquait l'importance de l'enterrement du placenta dans la culture polynésienne traditionnelle, mais aussi celle du traitement du cordon ombilical (taura pito) reliant le placenta au nombril (pito) de l'enfant; la question posée était celle de l'existence d'une symbolique intrinsèque à chacun de ces objets - outre les hommes, les îles étant également dites posséder un pito (nombril) - ou bien du sens qu'ils acquièrent par le rituel.

Le présent article souligne à nouveau l'attachement traditionnel des Tahitiens pour le rite - privé - d'enterrement du placenta, auquel fait écho celui - collectif - de fondation d'une église ou temple, dans un processus d'interaction. Pour une appréhension diachronique globale de ce sujet, il serait logique de lire d'abord le texte de 2002, puis celui-ci, et enfin celui de 2000.

17. Il convient bien sûr de lire, « le cordon ombilical».

18. Le miro est l'arbre (Thespesia Populnea) dit « bois de rose».

19. D'après Teuira Henry (1962 : 142), qui ajoute : « Lorsqu'on construisait un fare-ia-manaha (maison de conservation des objets du culte, située devant le marae), on tuait un homme et on l'enterrait sous le poteau central pour assurer la stabilité de la construction ». La logique demeure la même. Voir aussi Ellis $(1972$ : 218) au sujet de l'île de Huahine.

20. Est-ce parce qu'il s'agit d'un corps mort qu'il arrive que le placenta soit enterré au même endroit qu'un défunt, c'est-à-dire, en général, en Polynésie orientale, devant la maison familiale ? À l'inverse, faut-il pousser l'interprétation plus loin et considérer que c'est en tant que matière pourvoyeuse de vie que le placenta trouve sa place aux côtés des morts, voire dans cette juxtaposition de la matière ayant nourri le fotus et des ossements des morts dont les ossements possèdent euxmêmes une symbolique ambiguë ? Comme le rappelle Françoise Douaire-Marsaudon (2001), commentant des propos de Françoise Héritier pour les appliquer au cas polynésien, « dans de nombreuses cultures, la notion d'ancestralité est fondée sur 
En Polynésie orientale, c'est aux îles Australes que ces deux types de pratiques ont continué à se perpétuer jusqu'à une période relativement récente. Une sage-femme tahitienne atteste ainsi avoir vu en 1960 à Rapa, le placenta d'un nouveau-né enterré à même le sol dans une des dernières maisons traditionnelles en matériaux végétaux, maison basse ayant par ailleurs la forme avérée d'un sexe féminin. De nos jours, dans l'ensemble des cinq îles Australes, le placenta des enfants est enterré non pas à l'intérieur mais à l'extérieur, à l'entrée des maisons d'habitations ${ }^{21}$; et à Ra'ivavae (dans le même archipel), les morts sont encore mis en terre, tantôt à proximité immédiate des maisons, tantôt sous la dalle des terrasses, parfois à l'intérieur des habitations ${ }^{22}$. Une pratique identique était également observable à Rurutu où, au début du $\mathrm{Xx}^{\text {ème }}$ siècle, plusieurs personnes choisirent de se faire enterrer sous le plancher de leur maison, dans le village de Moera'i. Ceci n'a rien de surprenant si l'on se remémore la continuité entre les maisons et les marae bien notée par Pierre Vérin dans cette île ${ }^{23}$. Ce dernier rapporte aussi qu'à l'époque de ses recherches (1962), le cordon ombilical, était " placé à l'intérieur d'une coquille de turbo ('apu pūpū). Il s'agit sans doute d'une survivance des temps classiques : les relevés archéologiques ont révélé la présence de cistes de dalles de corail contenant des coquilles de turbo. Ces cistes étaient rien moins que des tanura'a pito (lieu d'inhumation du cordon ombilical) pour les enfants des ari'i (chefs). Sur le site Teh 3 et la terre [...], on a noté deux pierres levées sur lesquelles on prétend que ces cordons étaient sectionnés » $(1969: 271)^{24}$.

L'association du cordon ombilical avec la coquille du gastéropode turbo est pleine de sens ${ }^{25}$. Si, autrefois, le turbo contenant le cordon était fréquemment enterré sous les dalles d'une maison ou d'un marae, il pouvait aussi - et cette coutume s'est perpétuée aux îles Australes être jeté au large. Dans le cas où le cordon ombilical serait immergé - sa descente dans l'océan étant perçue comme un gage de souffle pour l'enfant -, c'est alors le placenta (püfenua) qui est mis en terre au seuil de la maison. Le plus étonnant cependant est que les Polynésiens des Australes qui perpétuent pour partie ces rites, placent aujourd'hui de préférence le cordon ombilical de l'enfant dans un petit bocal que le père remplira d'eau pour qu'il coule en profondeur, tel le coquillage utilisé autrefois. Et si d'autres choisissent d'enterrer à la fois le placenta et le cordon ombilical à l'entrée de leur habitation, ils enterrent souvent ce cordon dans le bocal qui le renferme hermétiquement, rappelant une nouvelle fois la pose du bocal à l'intérieur du niu d'un édifice chrétien.

$\mathrm{Au}$ terme de cette étude, nous avons pu mettre en évidence les nombreuses similitudes existant entre les rites liés au corps de l'homme et les

la présence d'une substance transmissible stockée dans les os, sur une semence dans laquelle se trouve le principe de transmission de la vie».

21. Poniari'i et Te'aue Tuheiava-Tavaeari'i rapportent qu'il en était de même à Maupiti jusqu'à il y a peu. Pour eux, la généralisation de la mise en terre d'un arbre sur le placenta est récente, sa destination leur semblant davantage être le seuil de la maison.

22. La coutume d'enterrer les morts, en particulier les enfants morts et éventuellement les fœtus, au seuil ou à l'intérieur des maisons est assez répandue dans le monde. Xavier Yvanoff (1998: 255-257) y consacre des passages intéressants de son volumineux ouvrage, hélas dépourvu de la référence des textes de base utilisés. D'une façon générale, de l'Inde à la Russie en passant par la Méditerranée, le fait pour les femmes de marcher à cet endroit favorisait une grossesse, tout comme « la coutume qui consiste à se rendre sur la tombe d'un saint homme afin de recueillir l'âme du défunt, ou d'être fécondée par le mort [...] très à l'honneur dans le monde juif et musulman et particulièrement dans toute l'Afrique du Nord » (idem : 257).

23. «L'utilisation de l'extrémité de la plate-forme d'une maison pour constituer la cour [du marae] est courante pour les marae secondaires ». (Vérin, 1969 : 286). Teuira Henry (1962:158) livre aussi un chant tahitien des marae faisant état de ce que la maison d'un homme important - ta'ata mana - était son marae, dans le sens d'espace sacré, et établissant une correspondance entre la porte de devant de la maison - 'iriāputa mиa ō te fare - et le mur du devant - ahu mua - du marae.

24. On trouve une indication importante, allant dans le même sens, dans les mémoires de Marau Ta'aroa (1971 : 50). Selon elle, le cordon ombilical de Turi Teri'itinorua, fils du dieu créateur Ta'aroa, fut détaché sur le marae Te ra'i maruarua, ce qui eut pour effet de rendre sacrés les marae royaux de ces îles. Or, Turi est le premier ari'i (chef) humain de la descendance des dieux. La section de son cordon ombilical sacralise certes les marae, mais elle inaugure aussi une ère nouvelle entre le temps des dieux et le temps des hommes.

25. Ce lien entre le cordon ombilical (pito) et le coquillage turbo (mä'oa) semble s'exercer doublement. Ici, le turbo sert d'enveloppe solide, de contenant au cordon, notamment à des fins d'immersion, mais d'une façon plus large, dans l'ordre de la culture tahitienne traditionnelle, c'est un rapport de fertilité que le turbo entretient avec le cordon ombilical : Teuira Henry rapporte ainsi (1962 : 402), que « la coquille de maoa (turbo) était enveloppée dans de la fougère maire (Polypodium pustulatum) et accrochée au-dessus d'une truie prête à mettre bas, pour donner force et vigueur aux petits ». Cette fougère maire " était tenue pour sacrée et comme ayant une influence bénéfique, étant issue du cordon ombilical de Ro'o, messager du dieu Tane, qui poussa sur un arbre fara auquel il resta accroché » (Henry $1962: 150$ ). Ailleurs, (p. 384), elle indique que c'est le pandanus fara servant de support à la fougère maire qui serait né du cordon ombilical de Ro'o.

Ce rapport de fertilité ou fécondité découle vraisemblablement de l'abondance de ce genre de coquillages, les turbos formant de nombreuses colonies sur les récifs, au point qu'une famille nombreuse de Tahiti est toujours connue sous l'appellation $m \bar{a}$ 'oa 'ie'ie (terme désignant ici le coquillage en forme d'escargot poussant sur l'osier sauvage). 
grandes étapes de l'élévation d'un temple, en Polynésie comme ailleurs. Le niu, dont la pose correspond au premier des rites d'élévation $\mathrm{du}$ corps du temple, est ainsi, en quelque sorte, son nombril ou son cœur ${ }^{26}$, point central appelé à toujours exister, même après sa mort, à l'intérieur d'un nouveau corps. Il est son origine et sa mémoire, les reliques des fidèles ou des morts manifestant la continuité de l'édifice et la pérennité de la communauté qui s'y rattache.

Mais peut-être est-ce abusivement que nous avons jusqu'ici utilisé le terme rite pour rendre compte de ces pratiques. Si la pose de la première pierre d'un temple s'inscrit à l'intérieur de rites religieux, la mise en terre du placenta et les manipulations du cordon ombilical constituent-elles véritablement des rites?

Il convient tout d'abord de les décrire comme des actions privées, accomplies par une seule ou deux personnes (géniteurs, grands-parents de l'enfant). Elles les effectuent à l'abri du regard des autres, afin de ne pas risquer un détournement de ces reliques d'origine par un tiers susceptible de porter atteinte à l'intégrité de l'enfant en les utilisant en sorcellerie. D'où la variété des pratiques et des significations qui leur sont données par ceux qui les accomplissent sans les avoir véritablement apprises, chacun improvisant sur la base de ce qu'il a entendu dire de ses propres frères, sœurs ou parents. Néanmoins, on préférera parler de rite privé et non de simple pratique : les traitements réservés au placenta et au pito participent en effet de ces rites à propos desquels Pierre Smith (1979:.146) note qu'ils " répondent à une série de circonstances périodiques (et) forment système selon un axe syntagmatique " ${ }^{27}$, c'est-à-dire, à l'intérieur d'un cycle. Ce cycle est celui des stades de la vie d'une personne (naissance, baptême, circoncision des gar- çons, mariage, etc.), mais il peut aussi s'agir des étapes de la vie d'un temple.

Il n'est pas dans notre propos d'entreprendre ici un réexamen global de la définition du rite. Pierre Smith, qui attribue pour sa part une efficacité au rite, remarque que pour les Occidentaux, "l'inauguration d'un édifice public n'est qu'une manifestation symbolique », tandis que « le baptême d'un navire revêt déjà les caractéristiques du rite », car « il se passe réellement quelque chose à ce moment, une opération mystérieuse ou mystique qui ne se laisse pas réduire au symbolisme du geste »' (1979: 140) ${ }^{28}$. En suivant sa définition, on peut poser que les pratiques polynésiennes liées au placenta relèvent davantage de rites d'efficacité que de simples gestes symboliques, dans la mesure où l'avenir de l'enfant, ses qualités de sociabilité, etc., sont conditionnés par les circonstances précises du rite. En revanche, la pose de la première pierre d'un temple, qui leur répond en écho, remplit essentiellement une fonction de mémoire, à laquelle ne se limite pas le rite d'enterrement du placenta ${ }^{29}$.

L'exemple polynésien nous montre aussi comment se produisent des interactions entre des rites de fondation qui obéissent au départ à une même logique ${ }^{30}$. L'importance sociale du rite consistant à introduire des reliques dans un bocal placé d'ordinaire à l'entrée d'un temple s'explique parce qu'il fait écho, pour chaque personne, à l'enterrement du placenta à l'entrée des maisons ainsi qu'à l'insertion dans un bambou ou un coquillage des reliques de l'enfant destinées au marae ou à l'océan. Quant au rituel d'introduction du bocal dans la première pierre ou le niu d'un édifice religieux, il influence à son tour les pratiques d'enterrement du cordon ombilical et la manière dont celui-ci est immergé dans l'océan. Ainsi, non seulement les hommes

26. Par nombril ou cœur, nous signifions l'idée de centralité et d'origine. Le mot nombril est en fait plus approprié que celui de cœur. Si aucun terme tahitien ne décrit le niu, point de base d'édification d'un édifice religieux, comme étant véritablement un cœur (mäfatu) ou un nombril (tumu pito), la symbolique qui se rattache à cet objet l'apparente directement au nombril, au cordon ombilical, ou au placenta dans la culture tahitienne.

27. Par opposition aux « rituels occasionnels (qui) eux, répondent aux surprises du temps, c'est-à-dire à des circonstances qui ne sont prévisibles que d'un point de vue statistique. Ils forment système selon un axe de type paradigmatique, offrant plusieurs genres de réponses rituelles adaptées à divers cas » (ibid.).

28. Son approche est donc bien plus restrictive que celle de Claude Lévi-Strauss (1964: 343) qui inclut sous la même dénomination de rites les actions qui, « simultanément ou alternativement, offrent à l'homme le moyen, soit de modifier une situation pratique, soit de la désigner et de la décrire. Le plus souvent, les deux fonctions se recouvrent, ou traduisent deux aspects complémentaires d'un même procès ».

29. Il ne faut pas confondre la pose de la première pierre d'un bâtiment avec son inauguration, même si des similitudes peuvent (à nouveau) être repérées entre les deux cérémonies. Ainsi, lors de l'inauguration d'un temple récemment rénové, celui de Te'āvaro à Mo'orea, le 30 août 1997, la cérémonie débuta-t-elle par la mise en terre d'un plant d'arbre à pain, symbole d'arbre de vie pour les Tahitiens. Le nom de l'édifice n'est d'ailleurs autre que Fare hotu 'maison fruit'. Voir La dépêche de Tahiti, 01-09-1997: 4 .

30. Smith (1979: 145 et 147) note encore que « les différents rites associés à des circonstances relevant d'une même série tendent à former un système [...]. Plusieurs systèmes rituels coexistent généralement au sein d'une même culture [...]. Si les cultures développent inégalement et différemment leurs systèmes rituels, elles établissent aussi entre eux des liaisons complexes et diverses ». 
communiquent-ils par les rites, mais les rites communiquent-ils entre eux, dans une dynamique continue.

\section{RÉFÉRENCES}

BABADZAN, Alain, 1983. Une perspective pour deux passages. Notes sur la représentation traditionnelle de la naissance et de la mort en Polynésie, L'Homme, t. XXIII, no 3, pp. 81-99.

Cadousteau, Geneviève Ma'i ari'i, 1987. Généalogies des arii des îles de la Société. Pape'ete, Société des Études Océaniennes, $131 \mathrm{p}$.

DAvies, John,1851. A Tahitian and English Dictionary. Tahiti, The London Missionary Society Press. 314 p.

Douaire-Marsaudon, Françoise, 2001. D’un sexe l'autre. Le rituel du kava et la reproduction de l'identité masculine en Polynésie, L'Homme, 157, pp. 7-34.

Dragan, Radu, 1999. La représentation de l'espace de la société traditionnelle. Paris, l'Harmattan, $367 \mathrm{p}$.

Église Évangélique de Polynésie Française, 1991. Papa haamori a te Etaretia Evanelia no Porinetia Farani. Pape'ete, E.E.P.F. 395 p.

Ellis, William, 1972. À la recherche de la Polynésie d'autrefois. Paris, Musée de l'Homme, Société des Océanistes, $\mathrm{n}^{\circ}$ 25, 1972, 2 vol. 943 p. (édition originale 1831 et 1832 Polynesian Researches. London, Fischer).

Henry, Teuira, 1962. Tahiti aux temps anciens. Paris, Société des Océanistes, Musée de l'Homme, 722 p. (édition originale 1928 Ancient Tahiti. Honolulu, P.P. Bishop Museum).

HÉRITIER, Françoise, 1989. Parenté, filiation, transmission, in Le père, métaphores paternelles et fonctions du père. Paris, Denoël ("L'espace analytique »), pp. 107-115.
Hiro, Émile Teri'iero’o,1997. E parau nō Huahine, Ra'iatea 'oia 'o Havai'i i mūta'a ihora, Huahine, 'o tei parau-ato'a-hia 'o Mata'irea, in Fare Vana'a (éd.), Académie tahitienne, Hei pua ri'i II, Pape'ete, 224 p. (pp. 30-33, 105-119 et 125-141).

LÉVI-STRAuss, Claude, 1964. Mythologiques. Le cru et le cuit. Paris, Plon, $402 \mathrm{p}$.

Mauss, Marcel, 1969. Essais de sociologie. Paris, Les éditions de minuit, 3 vol.

Marau Ta'aroa, 1971. Mémoires de Marau Taaroa, dernière reine de Tahiti, traduits par sa fille la princesse Takau. Paris, Musée de l'Homme, Société des Océanistes, $294 \mathrm{p}$.

Robineau, Claude, 1986. Taputapuatea, in Christian Gleizal (éd) Encyclopédie de la Polynésie, Pape'ete, vol. 4, pp. 94-95.

SAURA, Bruno, 2000. Le placenta en Polynésie française : un choix de santé publique confronté à des questions identitaires, Sciences sociales et santé, vol. $18, \mathrm{n}^{\circ} 3$, pp. 5-28.

- 2002. Continuity of Bodies : the Infant's Placenta and the Island's Navel in Eastern Polynesia, The Journal of the Polynesian Society, vol. 111, $\mathrm{n}^{\circ} 2$, pp. 127-145.

SмIтH, Pierre, 1979. Aspects de l'organisation sociale des rites, in Izard, Michel et Pierre Smith (sous la direction de). La fonction symbolique. Essais d'anthropologie. Paris, Gallimard, pp. 139-170.

Stimson, J. Frank et Donald Marshall, 1964. A Dictionary of some Tuamotuan dialects of the Polynesian language, Peabody Museum, Salem, Massachusetts et La Hague, 623 p.

Van GenneP, Arnold, 1909. Les rites de passage, Paris, Émile Nourry (réédition 1981, A. et J. Picard), $288 \mathrm{p}$.

VÉRIN, Pierre, 1969. L'ancienne civilisation de Rurutu (îles Australes - Polynésie française). La période classique. Paris, O.R.S.T.O.M., 318 p.

Yvanoff Xavier, 1998. Mythes sur l'origine de l'homme, Paris, Errance. 